\title{
Uniform Induced Electromagnetic Features around Longitudinal and Transverse Cracks
}

\author{
Jiuhao Ge, Wei Li*, Yanyun Wu and Xinan Yuan \\ Centre for Offshore Engineering and Safety Technology, China University of Petroleum (East China), Qingdao, China \\ *Corresponding author
}

\begin{abstract}
Uniform alternating electromagnetic field will be induced in specimen with alternating current excitation. The distribution of induced field will be perturbed by the existence of crack. To study the perturbation feature caused by crack, the specimen containing longitudinal and transverse cracks are detected in the experiment in this paper. The experiment indicates that the longitudinal and transverse cracks can be both detected out in one scan. Furthermore, the simulation of steel and aluminum with longitudinal and transverse cracks are carried out to explain the phenomenon. The result in simulation indicates that while longitudinal and transverse cracks exist in the surface of specimens, the induced alternating magnetic field around the crack would leak out of the specimen, meanwhile, the induced alternating current field around the crack would be disturbed and generated perturbation magnetic field. The magnetic field signal picked above the crack included the leakage magnetic field signal and perturbation magnetic field signal.
\end{abstract}

Keywords- alternating electromagnetic field; ACFM; AC-MFL; longitudinal and transverse cracks

\section{INTRODUCTION}

The uniform alternating electromagnetic field will be induced in ferromagnetic specimen with alternating current excitation [1-2]. And the alternating current field measurement (ACFM) and alternating current magnetic field (AC-MFL) are both techniques which used to detect cracks in structure using alternating electromagnetic field. However they are different in measurement and analysis of the resultant magnetic field perturbations caused by cracks [3]. In ACFM technique, the magnetic field caused by perturbation current field is measured to detect crack, but in AC-MFL technique the leakage magnetic field measured is used to predict the metal condition [4-5]. The theory of ACFM and AC-MFL are shown in Figure 1 and Figure 2.

A considerable amount of literature has been reported that ACFM and AC-MFL techniques are usually applied to detect transverse and longitudinal cracks respectively [6-10]. However, according to the research, the circumferential and axial defects could be inspected with the same magnet and sensor configuration using the AC-MFL [11-12]. Because sine excitation causes eddy current, the MFL field and disturbance eddy current field in the defect area of a conductor may lead to the confusion in the detection mechanism which was not primarily originated from AC-MFL effect using a U-shape inducer to detect longitudinal crack [13]. However, sporadic literatures have involved the research of combined action of magnetic field and current field around crack.

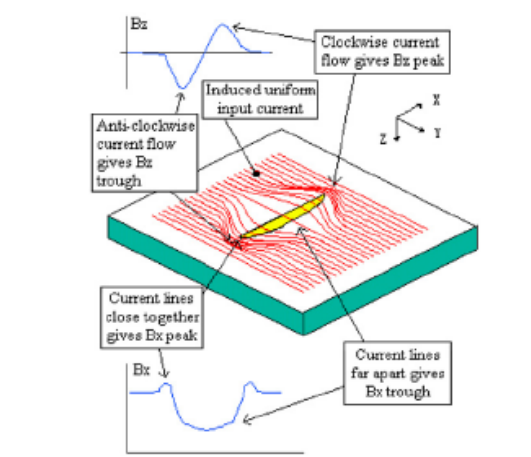

FIGURE 1. THE THEORY OF ACFM

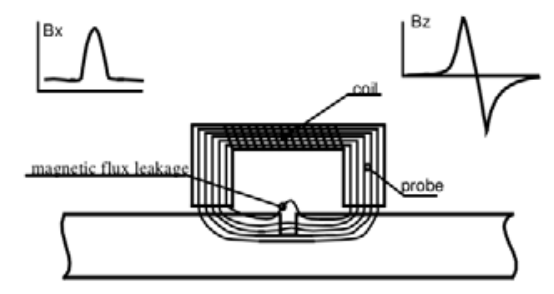

FIGURE II. THE THEORY OF AC-MFL

To research the distribution feature of alternating electromagnetic field around crack, the experiment and simulation involving specimens of ferromagnetic and nonferromagnetic material with longitudinal and transverse cracks will be carried out in this paper. The results in this paper would lay the foundation for the detection of crack using acelectromagnetic field.

\section{EXPERIMENT}

\section{A. Experimental System}

The experiment was carried out to verify the discovery reported in the literatures [11-12] by accident. The ac electromagnetic field detection experimental system was set up, as seen in Figure 3, and The u-shape probe was excited by an alternating current source of $\mathrm{I}=1 \mathrm{~V}$ and $\mathrm{f}=1000 \mathrm{~Hz}$, as shown in Figure 4. The output of the detection sensor was filtered, amplified and then digitized using $\mathrm{A} / \mathrm{D}$ acquisition. In the end, the test results, $B_{\mathrm{x}}$ and $B_{\mathrm{z}}$, were showed in the PC. 


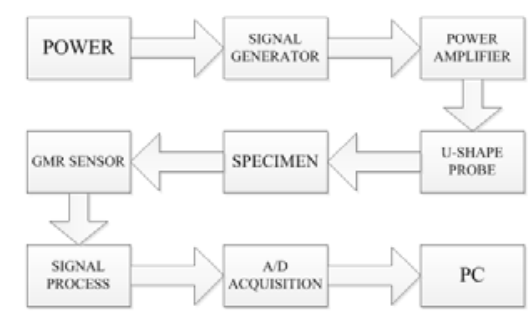

FIGURE III. AC ELECTROMAGNETIC FIELD DETECTION SYSTEM

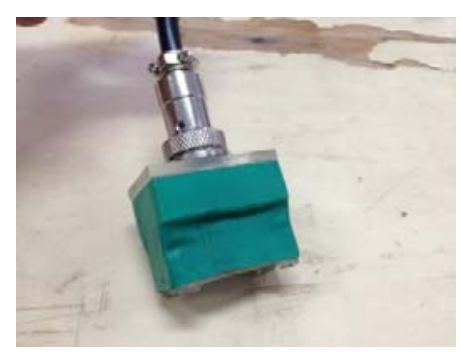

FIGURE IV

AC-ELECTROMAGNETIC PROBE

\section{B. Experiment Results}

The steel specimen with longitudinal and transverse cracks was used with dimensions $\mathrm{L}=50 \mathrm{~mm}, \mathrm{~W}=2 \mathrm{~mm}, \mathrm{P}=5 \mathrm{~mm}$, as shown in Figure 5. The experiment results are shown in Figure 6 and Figure 7.

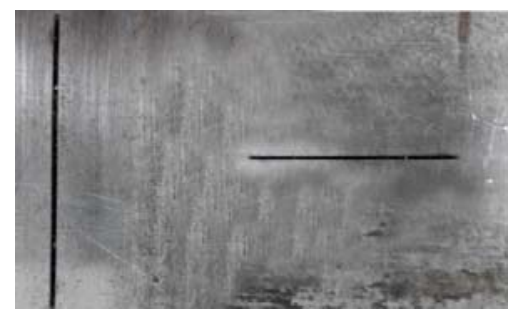

FIGURE V.

SPECIMEN WITH LONGITUDINAL AND TRANSVERSE CRACKS

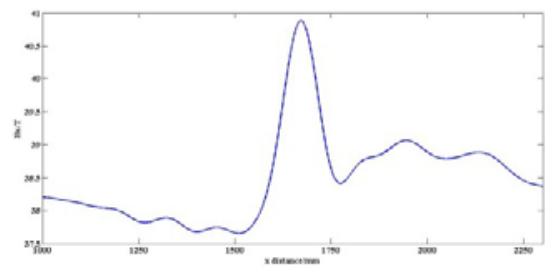

$\mathrm{a}$

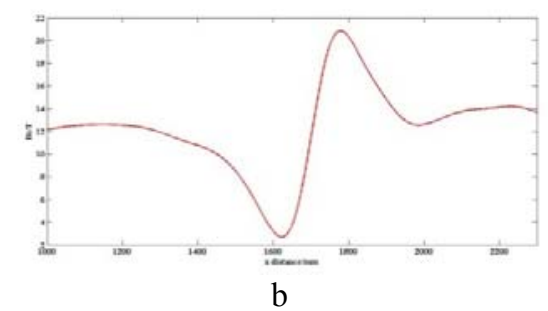

a) $B_{x}$ signal b) $B_{z}$ signal

FIGURE VI.

LONGITUDINAL CRACK RESULT

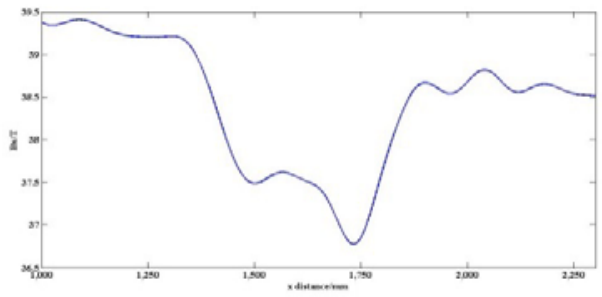

$\mathrm{a}$

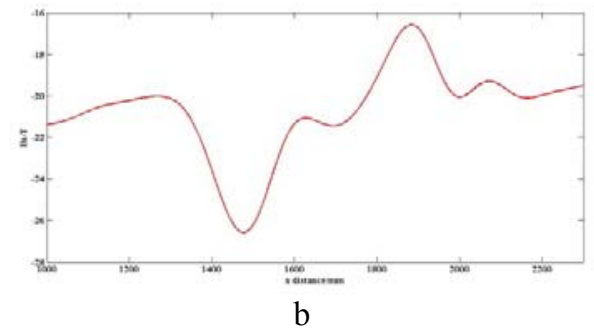

a) $B_{\mathrm{x}}$ signal b) $B_{\mathrm{z}}$ signal

FIGURE VII. TRANSVERSE CRACK RESULT

It is apparent from these figures that the longitudinal and transverse cracks could be detected out actually which match the result in literatures using the ac excitation in one scan. Analyzing the $B_{\mathrm{x}}$ signals, we could distinguish the longitudinal and transverse crack.

\section{SIMULATION}

To explain the phenomenon discovered in the experiment further, the 3D FEM model was built with the COMSOL Multiphasic, as shown in Figure 8. The model parameters were shown in Table 1. The magnetic fields physics and frequency domain analysis were used in the simulation. To save calculation time, the impedance boundary was applied to the specimen surface.

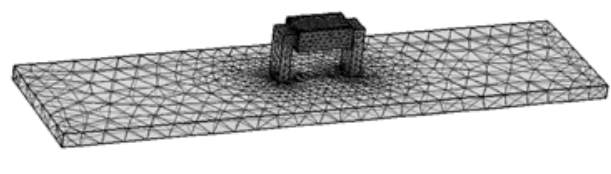

FIGURE VIII. 3D FEM MODEL

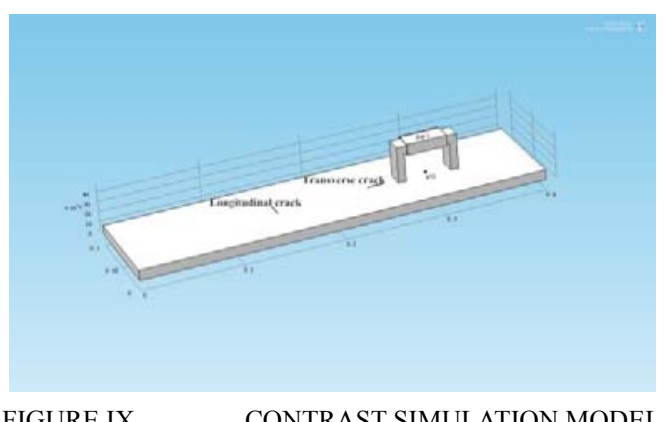

FIGURE IX. CONTRAST SIMULATION MODEL 
TABLE I.

SIMULATION PARAMETERS

\begin{tabular}{cc}
\hline NAME & VALUE \\
\hline EXCITATION COIL & 6KHz, 1A, 500TURNS \\
SPECIMEN & STEEL $\Sigma=1.12 \mathrm{E} 7 \mathrm{~S} / \mathrm{M}, \mathrm{M}_{\mathrm{R}}=1000$ \\
ELEMENTS & 49695 \\
CRACK & LENGTH $=10 \mathrm{MM}$, WIDTH $=0.8 \mathrm{MM}$, \\
& DEPTH=3MM
\end{tabular}

The AC-MFL could only be used to detect ferromagnetic material [6-7]. To research the ac-electromagnetic field features around cracks, steel and aluminum specimens were detected respectively. Moreover, the longitudinal and transverse cracks were placed in the specimen surface as shown in Figure 9.

The results obtained from the analysis could be compared in Figure 10 and Figure 11. From the data in Figure 10 and Figure 11, it is apparent that when interrogating steel specimen, the longitudinal and transverse cracks could be detected out as the result in experiment indicating the correctness of simulation model. When interrogating aluminum specimen, only the transverse crack could be detected out distinctly. Because of the low permeability, there is only perturbation magnetic field existence caused by perturbation current in the tips of the crack when detecting cracks in aluminum specimen. Moreover, the variation of $B_{\mathrm{x}}$ and $B_{\mathrm{z}}$ are much smaller than the signals in steel specimen. Then, there is larger background magnetic field than the steel in $\mathrm{x}$ direction.

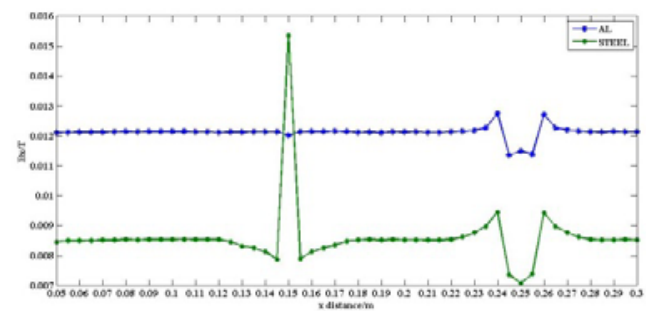

FIGURE X.

$B \times$ IN STEEL AND ALUMINUM SPECIMEN

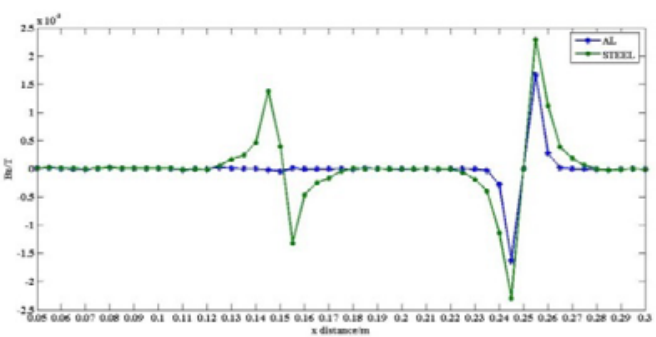

FIGURE XI.

BZ IN STEEL AND ALUMINUM SPECIMENTo prove the existence of the perturbation magnetic field when detecting longitudinal crack, another aluminum and steel specimen with cross crack were interrogated, as shown in Figure 12.

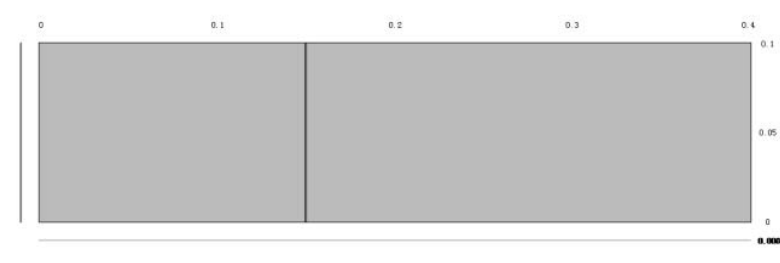

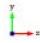

\section{FIGURE XII. CROSS CRACK}

Figure 13 and 14 compare the results obtained from simulation. What is interesting in Figure 13 and Figure 14 indicates that when the length of crack is as long as specimen width, there is no current disturbed in the tips of the crack. The variation of $B_{\mathrm{x}}$ in aluminum specimen is zero. Only the crack in steel specimen could be detected out. Comparing the Figure 10 and Figure 13, it is clear that there is bigger variation of $B_{x}$ signal in Figure 10, when detecting longitudinal crack in aluminum. Thus, the magnetic signal picked when detecting longitudinal crack in aluminum is caused by perturbation current field.

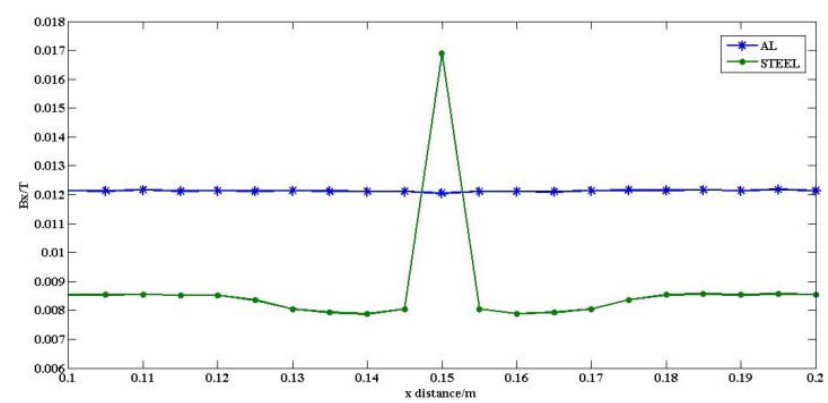

FIGURE XIII. $\quad B \times$ IN STEEL AND ALUMINUM SPECIMEN

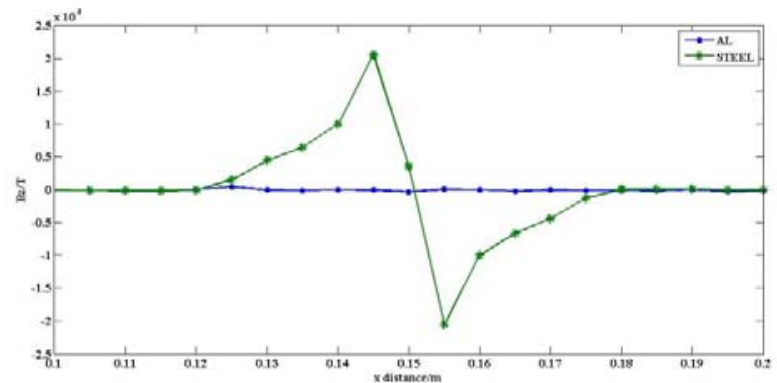

FIGURE XIV. Bz IN STEEL AND ALUMINUM SPECIMEN

Strong evidence is found that the leakage magnetic field and the perturbation magnetic field are existence simultaneously around cracks in steel specimen of high permeability and conductivity.

The analysis showed that when detecting ferromagnetic specimen using AC excitation, the ac-electromagnetic field were induced in the specimen. While longitudinal and transverse cracks existed in the surface of specimens, the 
induced alternating magnetic field around the crack would leak out of the specimen, meanwhile, the induced alternating electrical field around the crack would be disturbed and generated perturbation magnetic field. The magnetic field signal picked above the crack included the leakage magnetic field signal and perturbation magnetic field signal.

\section{CONCLUSION}

Prior studies [11-12] that have noted circumferential and axial defects could be inspected with the same magnet and sensor configuration using the ac electromagnetic field. However, there was no appropriate explanation about these results.

The present study was designed to determine the acelectromagnetic field features for crack. We have carried out experiment to detect the longitudinal and transverse cracks in one scan. Furthermore, simulations of steel and aluminum specimens containing longitudinal and transverse cracks were also researched. It can be concluded by the following:

(1) In the experiment, it was apparent from these results that the longitudinal and transverse cracks could be detected out in one scan.

(2) When detecting the ferromagnetic specimen using AC excitation, the ac-electromagnetic field was induced in the specimen. While crack existed in the surface of specimens, the induced alternating magnetic field around the crack would leak out of the specimen, meanwhile, the induced alternating electrical field around the crack would be disturbed and generated perturbation magnetic field.

The results of this study supported the idea that the magnetic field $в$ around cracks was consisted of the leakage magnetic field ${ }_{B_{1}}$ and perturbation magnetic field ${ }_{B_{p}}$ in the acelectromagnetic field. And the feature of electromagnetic field around crack was analyzed. The conclusion in this paper would lay the foundation for the crack of arbitrary angle detection with alternating current excitation.

Further research should be done to investigate the theoretical equation on magnetic field caused by the arbitrary direction crack in the ac-electromagnetic field. This is an important issue for future research that estimating the crack angle in one scan.

\section{ACKNOWLEDGMENT}

This work is supported by the National Natural Science Foundation of China (No. 51574276), the Shandong Provincial Natural Science Foundation, China (No. ZR2015EM009), the Fundamental Research Funds for the Central Universities (No.15CX05024A), the Postgraduate Innovation Project of China University of Petroleum (No. YCX2015039), the Applied Basic Research Program of Qingdao City, China (142-4-49-jch).

\section{REFERENCES}

[1] J. Zhou, M C Lugg, R. Collins, "A non-uniform model for alternating current field measurement of fatigue cracks in metals," International Journal of Applied Electromagnetics \& Mechanics, 1999, 10(3):221-235.

[2] R. K Amineh, M. Ravan, S. H H Sadeghi, R Moini. "Using ac field measurement data at an arbitrary liftoff distance to size long surface- breaking cracks in ferrous metals." Ndt \& E International, 2008, 41(3):169-177.

[3] M. H Ostovarzadeh, "Analytical solution for interaction of an arbitrary frequency curved rectangular inducer with a transverse ring shaped groove surrounding a long conductive cylinder," Ndt \& E International, 2015, 69:55-63.

[4] G. Katragadda, W. Lord, Y. S Sun, S. Udpa, L.Udpa, "Alternative magnetic flux leakage modalities for pipeline inspection," IEEE Transactions on Magnetics," 1996, 32(3):31-31(1).

[5] A. H Salemi, S. H H Sadeghi, R. Moini, "Thin-skin analysis technique for interaction of arbitrary-shape inducer field with long cracks in ferromagnetic metals," Ndt \& E International, 2004, 37(6):471-479.

[6] Y. Gotoh, N. Takahashi, "Evaluation of alternating flux leakage testing using 3D nonlinear eddy current analysis and experiment," Electrical Engineering in Japan, 142, 1(2003), 8-15.

[7] Y. Gotoh, N. Takahashi, "Study on problems in detecting plural cracks by alternating flux leakage testing using 3D nonlinear eddy current analysis," Magnetics IEEE Transactions on, 2003, 39(3):1527 - 1530.

[8] M. Ravan, S. H H Sadeghi, R. Moini, "Field distributions around arbitrary shape surface cracks in metals induced by high-frequency alternating-current-carrying wires of arbitrary shape," Magnetics IEEE Transactions on, 2006, 42(9):2208 - 2214.

[9] A. M Lewis, D. H Michael, M. C Lugg, R Collins, “Thin - skin electromagnetic fields around surface - breaking cracks in metals," Journal of Applied Physics,” 1988, 64(8):3777-3784.

[10] L. Wei; C. Guo-ming; L. Feng; L. Zhun, "Study on ACFM crack angle detection with 1-D array probe," In Measuring Technology and Mechatronics Automation (ICMTMA), 2011 Third International Conference on, vol.3,417-419, 6-7 Jan. 2011

[11] C. K Low, B. S Wong, "Defect evaluation using the alternating current field measurement technique," Insight, 2004, volume 46(10):598-605(8).

[12] Z. S. Lim;D. R. Son, "Inspection of defects on the roll surface using ac magnetic flux leakage method," World Conference on Non-Destructive Testing 15th, 2000.

[13] K. Song, C. Chen, Y. Kang, J. Li, J Ren, "Mechanism study of AC-MFL method using U-shape inducer," Chinese Journal of Scientific Instrument”, 2012. 